\title{
Eine Antwort auf die Zunahme von HIV und andern Geschlechtskrankheiten bei MSM
}

\author{
In den letzten Jahren haben die Inzidenzraten für HIV, Syphilis und andere \\ Geschlechtskrankheiten bei MSM (Männern, die Sex mit Männern haben) be- \\ denklich zugenommen. Zudem zeigt sich bei MSM im Vergleich zu hetero- \\ sexuellen Männern allgemein eine schlechtere Situation bezüglich physischer \\ und psychischer Gesundheit. Mit einem schwulenspezifischen Gesundheits- \\ angebot reagieren die Kooperationspartner Zürcher Aids-Hilfe und ARUD \\ Zürich auf diese Entwicklungen.
}

Philip Bruggmann

Korrespondenz:

Philip Bruggmann

Leiter Somatik ARUD Zürich

Sihlhallenstrasse 30

CH-8026 Zürich

p.bruggmann@arud-zh.ch

\section{Hintergrund}

\section{HIV}

Die Zahl der HIV-Neuinfektionen nimmt bei MSM seit 2003 wieder zu, die neusten BAG-Zahlen für diese Risikogruppe sind alarmierend [1]. 2003 wurden 157 HIV-Erstdiagnosen bei MSM gestellt, 2006 waren es rund 300. Sex mit Männern ist die Ursache für durchschnittlich etwa $40 \%$ der HIV-Infektionen bei Männern und - seit Anfang der neunziger Jahre - für ein Viertel aller HIV-Infektionen in der Schweiz.

Gemäss Studien des IUMSP Lausanne (Institut universitaire de médecin sociale et préventive) beträgt die Prävalenz bei unter 30jährigen MSM 2\%, bei den über 30jährigen sind es sogar rund 12\% [2]. Auch die Zürcher Men's Study (ZÜMS) stellte in der Zürcher Schwulenszene eine Prävalenz von $12 \%$ fest [3]. In sexuell aktiveren Szenen ist jeder Sechste Träger des Virus, wobei 3 von 9 MSM nichts von ihrer Infektion wissen.

Der Anteil der MSM, die über kein aktuelles HIV-Testresultat verfügen (Test weiter zurückliegend als 12 Monate), ist seit 1994 steigend (38\% vs. $48 \%$ ). Allerdings haben 2000 80\% der MSM schon mindestens einmal im Leben einen HIVTest gemacht. Damit gehören MSM zu den testfreudigeren sozialen Gruppen - trotzdem ergab die ZÜMS-Studie, dass nur 2 von 3 HIV-positiven MSM von ihrer HIV-Infektion wissen.

STI (sexually transmitted infections)

In der Schweiz wie auch in anderen westlichen Ländern beobachtet man in den letzten Jahren bei MSM eine Erhöhung der Infektionen mit se-

\section{Checkpoint Zurich: une réponse} à l'augmentation du VIH et des autres maladies sexuellement transmissibles chez les HSH

Ces dernières années, les taux d'incidence pour le $\mathrm{VIH}$, la syphilis et autres maladies sexuellement transmissibles ont considérablement augmenté chez les HSH (hommes ayant des rapports sexuels avec des hommes). De plus, par rapport aux hommes hétérosexuels en général, on relève une situation plus grave chez les HSH en matière de santé physique et psychique. Avec un programme de santé spécifique pour les homosexuels, les partenaires «Zürcher Aids-Hilfe» et «ARUD Zürich» réagissent de manière concrète à ces développements.

xuell übertragbaren Krankheiten wie z. B. Syphilis, Gonorrhö oder Chlamydien [4]. Das Projekt «Onsite-Syphilis-Test» in Zürich (Sommer 2005) hat ebenfalls einen klaren Anstieg der Syphilisinfektionen bei MSM aufgezeigt.

Die Wahrscheinlichkeit, sich mit HIV zu infizieren oder HIV weiterzugeben, steigt mit einer anderen STI-Erkrankung stark an.

Es gibt immer mehr Daten, die zeigen, dass bei Sexualpraktiken, bei denen Blut im Spiel ist, Hepatitis C übertragen werden kann. So ist die 


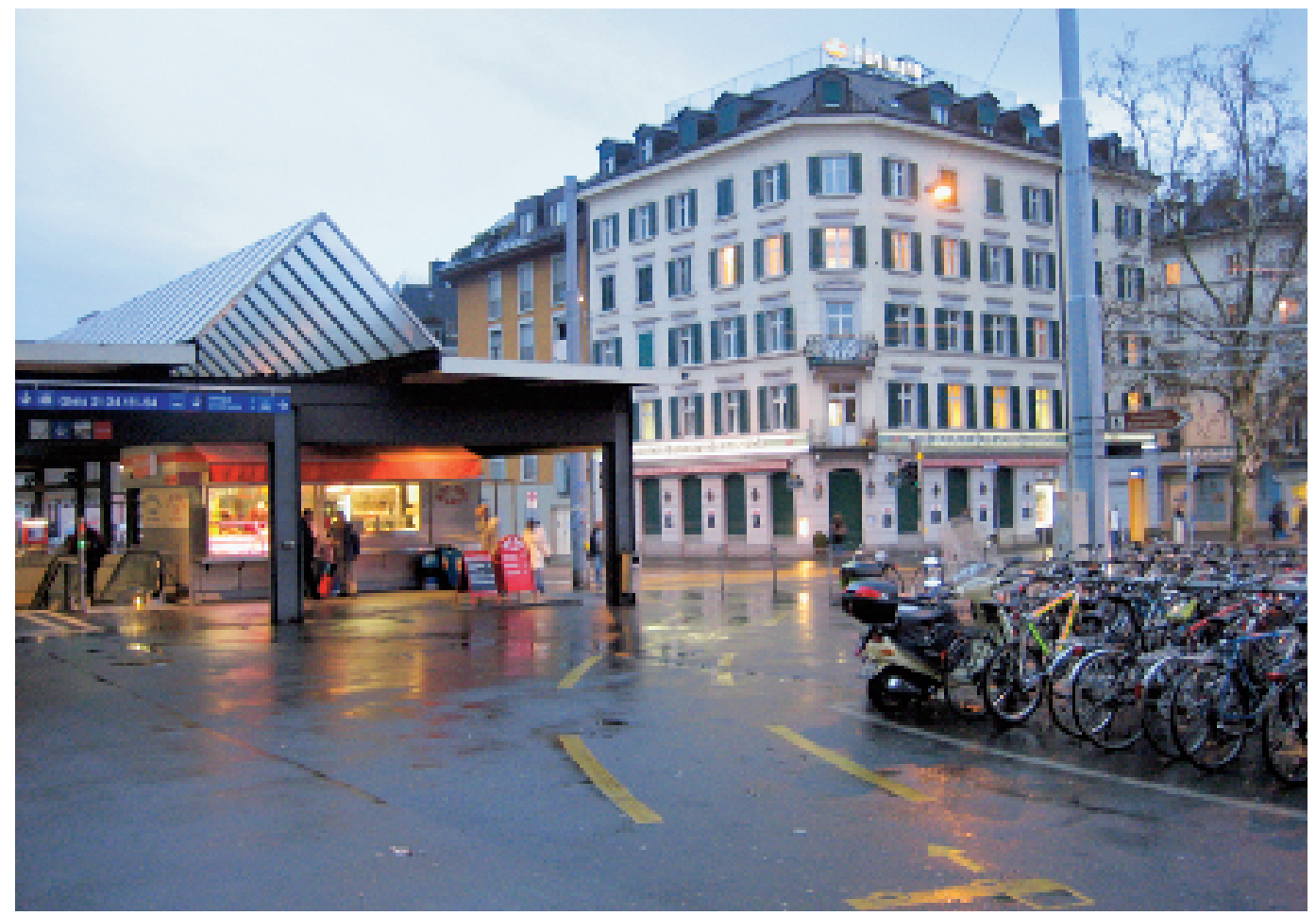

Zentraler Standort: Die Räumlichkeiten des Checkpoints (beleuchtete Fenster im dritten Stock) in unmittelbarer Nähe des Zürcher Hauptbahnhofs.

Hepatitis C bei MSM auch als STI zu sehen [5]. Ebenso Hepatitis A: Diese als Reisekrankheit bekannte Virushepatitis mit fäkooralem Übertragungsweg kann bei entsprechender Sexualpraktik auch sexuell übertragen werden.

\section{Allgemeine Gesundheit}

Die Santé-Gaie-Studie [6] zeigt in der Prävalenz von chronischen physischen Leiden wie auch von Angststörungen und Depression ein deutliches Ungleichgewicht zuungunsten der MSMGruppe im Vergleich mit der Allgemeinbevölkerung. Auch der Drogenkonsum ist in dieser Gruppe häufiger und ausgeprägter. Zudem beklagen die MSM deutlich häufiger eine Unzufriedenheit in der Beziehung zum behandelnden Hausarzt als die heterosexuellen Männer; viele MSM wünschen einen Arzt, der umfassend über Homosexualität und schwule Sexpraktiken und Bedürfnisse informiert ist. Eine diesbezüglich unverkrampfte Atmosphäre ist nötig für ein Gespräch über Sexualität und damit verbundene Krankheiten - und ein solches Gespräch mit dem Erstbetreuer ist Voraussetzung für die Erfassung von symptomlosen oder atypisch verlaufenden STI und deren Prävention.

\section{Zürich}

Zürich hat die grösste Schwulenszene der Schweiz. Viele MSM wählen Zürich als ihren
Lebensmittelpunkt: sei es wegen Arbeit oder Studium, sei es wegen der vielen schwulenspezifischen Ausgehangebote, mit und ohne Möglichkeiten für sexuelle Kontakte. Dies legt nahe, dass ein Grossteil der HIV- und STI-Infektionen bei MSM in Zürich stattfinden.

\section{Das Projekt Checkpoint Zürich}

Somit bestand in Zürich vordringlicher Handlungsbedarf gegen die steigenden Infektionszahlen in dieser Risikogruppe; Ziel ist es zudem, die allgemeine gesundheitliche Situation der MSM zu verbessern. Die Zürcher Aids-Hilfe (ZAH) beschloss, mit einem Gesundheitszentrum für MSM darauf zu reagieren.

Mit der ARUD Zürich fand die ZAH eine ideale Kooperationspartnerin (siehe Kasten). Aus der langjährigen Erfahrung in Drogenmedizin bringt die ARUD Zürich einerseits das nötige Know-how für infektiologische und allgemeinmedizinische Belange mit, anderseits auch das Wissen um den professionellen Betrieb von medizinischen Institutionen. Zudem konnten in der ARUD-Poliklinik GAIN die geeigneten, zentral beim Zürcher Hauptbahnhof gelegenen Räumlichkeiten und Infrastruktur angeboten werden [7].

Bestehende HIV-Testzentren im In- und Ausland werden häufig nach dem VCT-(VoluntaryCounselling-and-Testing-)Prinzip betrieben. Wie 
der Name sagt, geht es dort um Beratung und Tests. Meist ist das Testangebot auf HIV beschränkt. Wer eine Behandlung oder eine STI-Abklärung braucht, muss weitergewiesen werden. Dabei besteht immer die Gefahr des Kontaktabbruchs, insbesondere bei intimen Fragen wie der sexuellen Gesundheit.

Die Kooperationspartner ARUD Zürich und Zürcher Aids-Hilfe (ZAH) beschlossen daher, in ihrem Projekt «Checkpoint Zürich» neue Wege zu beschreiten, die über VCT hinausgehen: Durch die ärztlich geleitete Sprechstunde ist ein umfassendes Angebot möglich. So können nebst der Vor- und Nachtestberatung für HIV-Tests auch eine STI-Diagnostik, Impfungen und Therapien angeboten werden.

\section{Das Angebot}

So ist ein für Europa wohl einzigartiges Angebot entstanden, das spezifisch auf die diesbezügliche Risikogruppe MSM ausgerichtet ist. Beratungsgespräch, Arztkonsultation, HIV- und STI-Tests und entsprechende Behandlung und Nachkontrolle sind in ein und derselben Institution möglich. Die zentrale Lage neben den Perrons des Hauptbahnhofes ermöglicht eine gute Erreichbarkeit. Die Konsultationen können ohne Terminvereinbarung erfolgen. Die Beratung und Testung wird auf Wunsch auch anonym angeboten. Je nach Risikoanamnese werden HIVSchnelltests oder Kombinationstests (Antikörper und p24-Antigen) durchgeführt. Bei entsprechender Risikokonstellation und Einhaltung des Zeitfensters von maximal 72 Stunden besteht die Möglichkeit einer HIV-Postexpositionsprophylaxe (PEP). Alle Patienten werden auf virale Hepatitiden (A, B und C) getestet, je nach Immunlage werden Hepatitis A und B geimpft. MSM mit häufigem Partnerwechsel werden ein jährlicher Syphilistest und eine Kontrolle auf anale Kondylome (Präkanzerose) empfohlen. Mit jedem HIVTest wird gleichzeitig auch ein Syphilisscreening durchgeführt und umgekehrt.

\section{Erfahrungen aus 6 Monaten Checkpoint-Betrieb}

Anonyme HIV-Tests und die damit verbundenen Beratungsgespräche stiessen auf grosse Nachfrage. Im ersten halben Jahr der Betriebszeit verzeichnete Checkpoint Zürich 12 HIV-Neudiagnosen bei insgesamt 356 HIV-Schnelloder -Kombinationstests. Der Anteil an HIVNeudiagnosen ist vergleichsweise sehr hoch, er widerspiegelt aber noch nicht die geschätzte Prävalenz von $15 \%$ unter den sexuell aktiveren MSM. 211 Männer wurden in den ersten Monaten durch den Arzt behandelt.
Bei neu entdeckten HIV-Infektionen erfolgen die notwendigen weiteren Abklärungen sowie Aufklärungs- und Beratungsgespräche im Checkpoint Zürich. Im Anschluss werden die Patienten im Normalfall an die HIV-Sprechstunden der spezialisierten Praxen und Kliniken verwiesen. Die Durchführung einer HIV-Betreuung und allfälligen Therapie ist theoretisch im Checkpoint möglich, aber aufgrund der limitierten Öffnungszeiten nicht optimal und wird nur im Ausnahmefall auf speziellen Wunsch hin durchgeführt.

Viele Männer, die den Checkpoint aufsuchen, verfügen über keinen Hausarzt oder scheuen sich, sich mit ihren intimen Anliegen an diesen

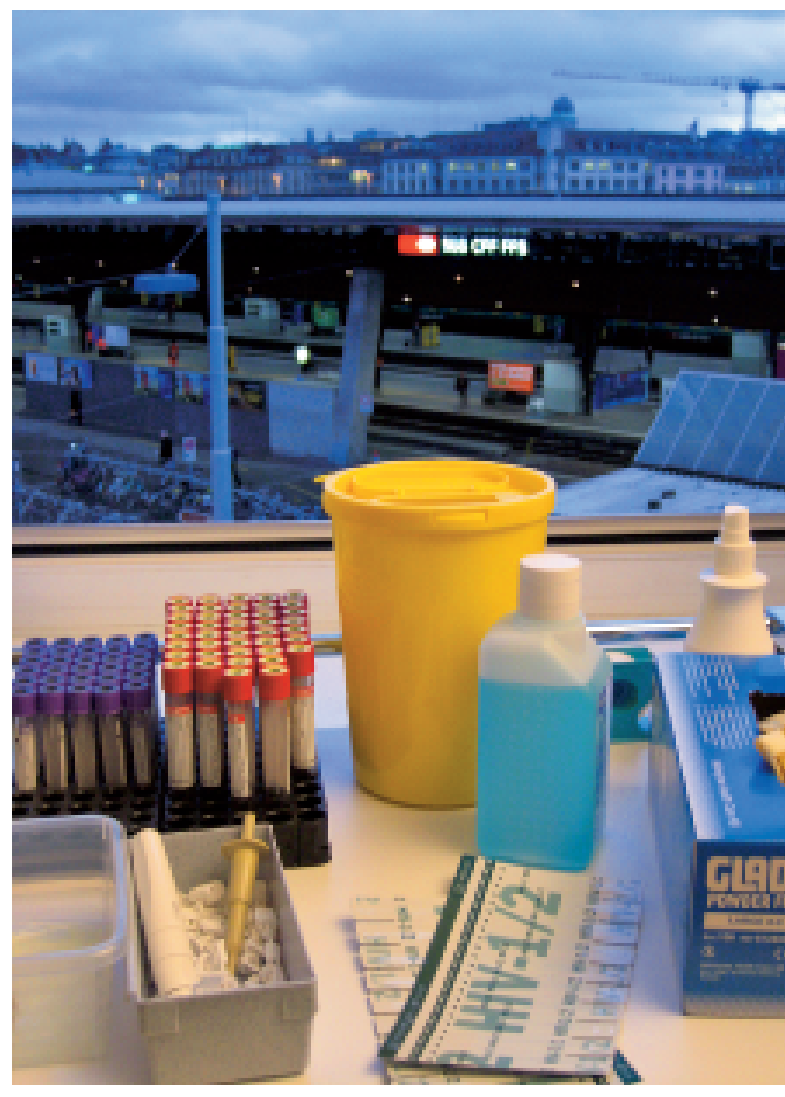

Das Angebot des Checkpoints umfasst Beratung, Abklärung und Behandlung.

zu wenden. Gerade Sexworker, die häufig aus Übersee stammen und sich nur temporär in der Schweiz aufhalten, sind medizinisch stark unterversorgt.

Nebst den klassischen Manifestationen der häufigsten STI Herpes, Gonorrhö und Chlamydien werden auch immer wieder orale und anale Formen dieser Geschlechtskrankheiten, die nicht dem «typischen Bild» entsprechen, entdeckt und behandelt. 
Allgemein erweisen sich die MSM in den Beratungsgesprächen als sehr gut informiert bezüglich der Risiken und der Prävention von HIV. Nicht selten kommt es aber in Situationen von Drogen- und Alkoholeinfluss zu einem Risikoverhalten, das nach Wiedererlangen der kognitiven Fähigkeiten bereut wird. Gerade in solchen Fällen wird die Durchführung einer Postexpositionsprophylaxe erwogen. Dabei kann mit einer der üblichen Dreierkombinationen von antiretroviralen Medikamenten über vier Wochen das Risiko einer allfälligen Ansteckung stark reduziert werden.

Das Wissen betreffend STI ist in der Szene weniger gut verankert, so dass sich bei der Beratungstätigkeit das Gespräch häufig um die verschiedenen Formen und Risiken der Geschlechtskrankheiten dreht. Eine Verminderung der STI-Prävalenz hat einen indirekten Einfluss auf die HIV-Prävention, begünstigen doch STI die HIV-Übertragung.

Patienten mit problematischem Drogenkonsum werden der ARUD-Poliklinik GAIN im selben Haus zugewiesen, wo eine spezifische, professionelle Beratung und Behandlung, abgestimmt auf das jeweilige Substanzproblem, angeboten werden kann [7].

\section{«Checkpoint mobile» und neue Öffnungszeiten}

Das Angebot der Checkpoint-Sprechstunde ist im ersten Betriebshalbjahr zunehmend häufiger benutzt worden. Die Sprechstunden an zwei Wochentagen reichen nicht mehr aus, um die Nachfrage zu bewältigen. Daher wird ab 1. Februar Checkpoint Zürich dreimal in der Woche seine Türen öffnen (siehe unten).

Um die MSM, die ihren Serostatus nicht kennen, aber ein hohes sexuelles Risikoverhalten aufweisen, besser zu erreichen bzw. um die Tests

\footnotetext{
ARUD Zürich (Arbeitsgemeinschaft für risikoarmen Umgang mit Drogen) Die ARUD Zürich ist ein 1991 gegründeter gemeinnütziger Verein, der heute im Raum Zürich vier Polikliniken für Drogenmedizin anbietet: Sowohl in den Polikliniken Zokl1, Zokl2 und DBB Horgen wie auch im medizinischen Zentrum GAIN besteht ein umfassendes psychiatrisches, medizinisch-infektiologisches, psychotherapeutisches und soziales Betreuungsangebot. Die ARUD Zürich beschäftigt knapp 100 Mitarbeitende, davon 17 Ärztinnen/ Ärzte, 4 Sozialarbeiter/innen und 8 Psychotherapeuten/-innen. Als privater Verein wird die ARUD Zürich nach unternehmerischen Gesichtspunkten geführt, die medizinischen Angebote werden finanziert durch Leistungsverträge mit den Krankenkassen. Die ARUD Zürich ist in der Forschung aktiv und engagiert sich für einen liberalen, medizinisch fundierten Umgang mit Drogen.
}

noch niederschwelliger anbieten $\mathrm{zu}$ können, wird Checkpoint in den einschlägigen Lokalen in Zürich mobile HIV- und Syphilistests mit entsprechender Beratung anbieten. Ziel von «Checkpoint mobile» ist es, Frischinfektionen mit hohem Übertragungspotential möglichst früh zu entdecken und die Weiterverbreitung bestmöglich einzudämmen. Dazu werden die geschulten Pflegefachmänner in Saunen, Clubs und Darkrooms in speziell eingerichteten Räumen Vortestberatungen und Blutentnahmen für HIV-Kombi- und Syphilisscreeningtests durchführen. Die Resultatmitteilung und die Nachtestberatung finden am darauffolgenden Werktag im Checkpoint an der Konradstrasse statt. Das ganze mobile Angebot erfolgt anonym.

\section{Checkpoint Zürich}

Konradstrasse 1, 8005 Zürich

(neben Hauptbahnhof). Tel. 0444555910

Öffnungszeiten:

Montag, Mittwoch und Freitag

von 16 bis $20 \mathrm{Uhr}$

Termine mit oder ohne Voranmeldung möglich

www.checkpoint-zh.ch

\section{Literatur}

1 Bundesamt für Gesundheit, Sektion AIDS. Die HIVEpidemie in der Schweiz gegen Ende 2006. BAG Bulletin. 2006;48:953-61.

2 Dubois-Arber F, Jeannin A, Meystre-Agustoni G, et al. Evaluation der HIV/Aids-Präventionsstrategien der Schweiz: Kurzfassung des siebten zusammenfassenden Berichtes 1999-2003. Lausanne: Institut universitaire de médecine sociale et préventive; 2003.

3 Wang J, Twisselmann W, Somaini B, Dubois-Arber F. HIV-Prävalenz und selbst mitgeteiltes Schutzund Risikoverhalten bei homosexuellen Männern in Zürich 1998. BAG Bulletin. 1999;49:916-9.

4 Klausner JD, Wong W. Sexually transmitted diseases in men who have sex with men: a clinical review. Curr Infect Dis Rep. 2003;5(2):135-44.

5 Cohen DE, Russel CJ, Golub SA, et al. Prevalence of hepatitis $\mathrm{C}$ virus infection among men who have sex with men at a Boston community health center and its association with markers of high-risk behavior. AIDS Patient Care STDS. 2006;20:557-64.

6 Häusermann M, Wang J. Projet Santé Gaie. Les premiers résultats de l'enquête sur la santé des hommes gais de Genève. Genève: Dialogai; 2003.

7 Stark L, Meili D. «GAIN - Gesundheitsangebot und Information» der ARUD Zürich: Veränderung des Drogenkonsums bedingt ein verändertes Angebot. Schweiz Ärztezeitung. 2006;87(35):1499-501. 